\title{
Intelligent Software Defined Atmospheric Effect Processing for 5th Generation (5G) Millimeter Wave (MMWave) Communication System
}

\author{
S. K. Agrawal ${ }^{\mathrm{a},}$, Kapil Sharma $^{\mathrm{b}}$ \\ ${ }^{a, b}$ Computer Engineering Department, Delhi Technological University (DTU), Formerly : Delhi College of \\ Enginering (DCE), Delhi, India
}

Received: 06 July 2017; Accepted: 17 January 2018; Published: 08 March 2018

\begin{abstract}
In this paper, we present atmospheric effect on 5th Generation (5G) millimeter wave (MMWave) communication system. Atmospheric effects for Delhi (India) based 5G communication system is calculated as per Delhi atmospheric conditions. Atmospheric impairments are major cause of degrading mmWave signal power while mmWave propagation in wireless channel. Due to Atmospheric impairments attenuation takes place and major impairments are like water vapour, oxygen, rain and fog for Delhi (India). 5G mmWave attenuation calculations are performed for the mmWave band frequencies $28 \mathrm{GHz}, 37 \mathrm{GHz}$ and $39 \mathrm{GHz}$. In this paper intelligent adaptive transmitter based on trend of the atmospheric conditions tunes to machine learning (ML) based derivation of channel capacity. The ML based transmitter is a supervised ML device and it has provision of self teaching learning machine based on data. Results are graphed for the mentioned frequencies and also intelligently software defined (SD) Shannon channel capacity calculated for Delhi (India) based 5G mmWave communication system under different atmospheric conditions.
\end{abstract}

Index Terms: Millimeter Wave (mmWave), 5th Generation (5G), Software Defined (SD).

(C) 2018 Published by MECS Publisher. Selection and/or peer review under responsibility of the Research Association of Modern Education and Computer Science

\section{Introduction}

The Worldwide continuous demand of data on wireless communication system having user ubiquitous access requires high speed data rate and high bandwidth to full the requirement seamlessly. Around $3 \mathrm{GHz}$

* Corresponding author. Tel.: +919910741162

E-mail address: sachin.ecin@gmail.com 
several research has been done to justify and provide the efficient data bandwidth and data rates; however this is not sufficient for upcoming years [1-7].

There is demand and need to fulfill this high speed data rate and high bandwidth along efficient frequency spectrum with intelligence in the system. 5th generation $(5 \mathrm{G})$ millimeter wave (MMWave) communication system is an emerging technology which will provide efficient mmWave spectrum utilization to provide high speed data rate and high bandwidth. MMWave frequencies bands are capable to provide the high speed data rate and high bandwidth by fulfilling the spectrum shortage. 5G MMWave communication system requires efficient spectrum handling by considering various MMWave propagation channel characteristics for the entire MMWave range. MMWave frequencies range from 30 to $300 \mathrm{GHz}$ and provide a large spectrum. It forms the base for the 5G MMWave communication system. In this paper, we propose a feature for an intelligent ML based adaptive transmitter for $5 \mathrm{G}$ wireless system [5-13].

\section{5G MMWave Frequency Spectrum}

High speed data rate and high bandwidth can be achieved under 5G communication system by having MMWave frequency spectrum. Several MMWave frequencies are proposed globally for 5G MMWave communication system. 5G MMWave frequency spectrum offers high speed data rate and high bandwidth. 5G communication system research is under development with MMWave frequencies. Recently; The United States Federal Communications Commission (FCC) authorizes and proposes broadband device operations in the following MMWave bands [13-16]:

- $27.5-28.35 \mathrm{GHz}$ band (28 GHz band)

- 37-38.6 GHz band (37 GHz band)

- 38.6-40 GHz band (39 GHz band)

FCC proposed MMWave bands are considering:

- High speed

- Low latency

- High capacity

- Advance user experience

\section{Atmospheric Gaseous Attenuation}

MMWave frequency link suffers with atmospheric attenuation and this MMWave attenuation takes place due to atmospheric gaseous absorption. The impact of atmospheric gases on the propagation of MMWave comes in form of:

- Signal attenuation

- Phase shift and

- Angle of arrival variations

Atmospheric attenuation takes place majorly due to atmospheric water vapor and atmospheric oxygen, scattering and scintillation. For certain MMWave frequencies losses are high because of the resonance gas molecules. Atmospheric water vapour attenuation is high as compare to oxygen particles due to atmospheric absorption and scattering [16-19]. 
(5G) Millimeter Wave (MMWave) Communication System

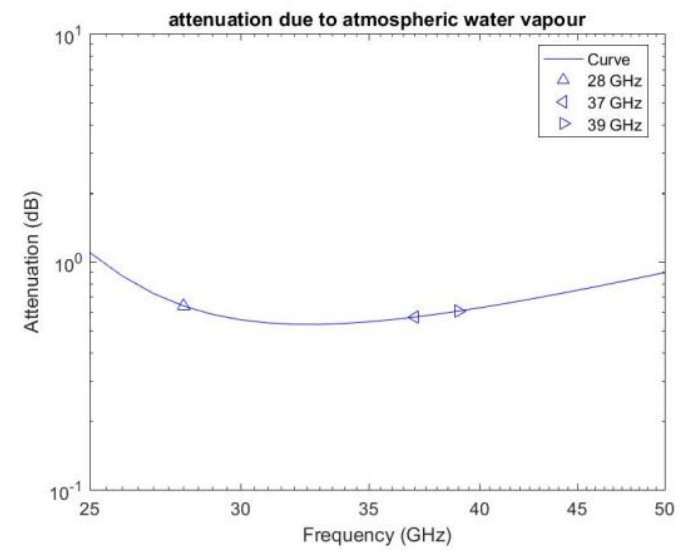

Fig.1. Atmospheric Water Vapor Attenuation for 5G mmWave Wireless Communication System

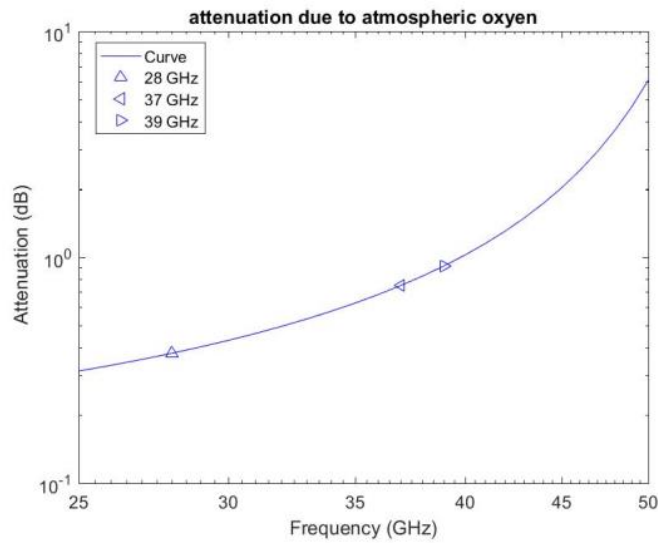

Fig.2. Atmospheric Oxygen Attenuation for 5G mmWave Wireless Communication System

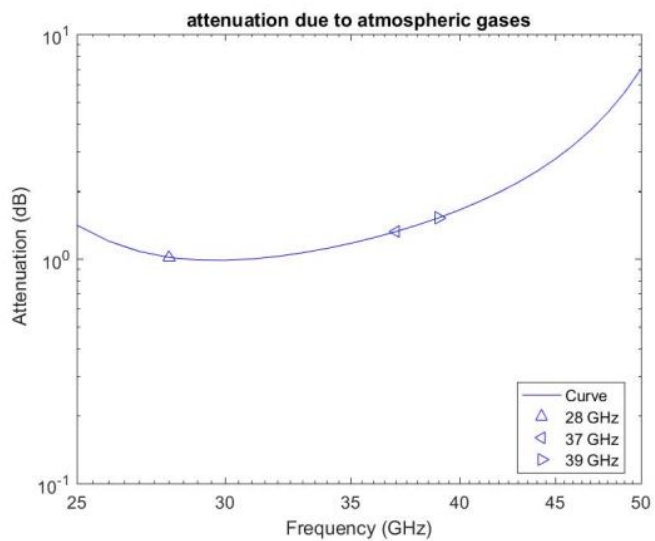

Fig.3. Atmospheric Gaseous Attenuation for 5G mmWave Wireless Communication System 
To calculate 5G MMWave communication system; atmospheric attenuation here we are using ITU-R recommended method for calculating atmospheric water vapor attenuation and atmospheric oxygen attenuation. This model comprises various parameters like; Frequency, Path-elevation angle, Height above mean sea level, Water vapor density and etc. The atmospheric gaseous attenuation is calculated by having equi probable summation of the atmospheric water vapor attenuation and oxygen attenuation [16-21].

Atmospheric water vapor specific attenuation is given by following equation (1):

$$
\gamma_{w}(f)=F(f)
$$

In equation (1) 'rho' is the water vapour concentration in $\mathrm{g} / \mathrm{m}^{\wedge} 3$ and ' $\mathrm{f}$ ' is the frequency in GHz. For Delhi rho changes from $6-10 \mathrm{~g} / \mathrm{m}^{\wedge} 3$. The total MMWave atmospheric water vapour attenuation is shown by the following equation (2)

$$
\begin{aligned}
A_{\text {watervapour }} & =\frac{h_{w} \gamma_{w}}{\sin \theta} \ldots \ldots \ldots \ldots \ldots \ldots \ldots \ldots \ldots \ldots . . . . . . . . . . . . . . .10 \\
A_{\text {watervapour }} & =\frac{\gamma_{w} \sqrt{R_{e} h_{w}}}{\cos \theta} F\left(\tan (\theta) \sqrt{R_{e} / h_{w}}\right) . . \theta \leq 10^{\prime \prime \prime}
\end{aligned}
$$

The details about above equations and related functions are explained the cited reference. As per equations (1) and (2) attenuation due to the MMWave water vapour has been plotted with mmWave frequencies in the Fig. (1). In this Fig. (1) logarithmic scale has been used with the MMWave frequency range $10 \mathrm{GHz}$ to $1000 \mathrm{GHz}$. Rho $=8$ for Delhi and as per plot in the Fig. (1) atmospheric water vapor shows high value attenuation due to resonances at $22 \mathrm{GHz}$. The variation of atmospheric attenuation due to the water vapour along with MMWave frequencies is shown in the Fig. (1). Due to high MMWave resonance frequency absorption mmWave attenuation takes place [16-21].

Atmospheric oxygen specific attenuation is given by the following equation (3)

$$
\gamma_{o}(f)=F(f)
$$

Atmospheric oxygen specific attenuation is given by the following equation (3) having MMWave frequency in GHz. The total attenuation in $\mathrm{dB}$ is given by the equation (4):

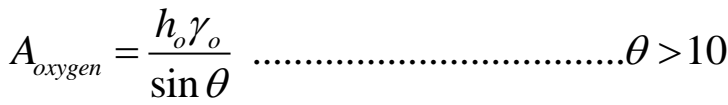

$$
\begin{aligned}
& A_{\text {oxygen }}=\frac{\gamma_{o} \sqrt{R_{e} h_{o}}}{\cos \theta} F\left(\tan (\theta) \sqrt{R_{e} / h_{o}}\right) . . \theta \leq 10
\end{aligned}
$$

As per equations (3) and (4) attenuation due to the MMWave oxygen has been plotted with mmWave frequencies in the Fig. (2). In this Fig. (2) logarithmic scale has been used with the MMWave frequency range $10 \mathrm{GHz}$ to $1000 \mathrm{GHz}$. Atmospheric oxygen shows high attenuation due to strong bands of resonances at 57-60 GHz. Fig. (2) shows graph of Delhi atmospheric attenuation due to oxygen. 5G MMWave communication 
system; MMWave transmission windows are at $35 \mathrm{GHz}$ and $94 \mathrm{GHz}$. These windows are having minimal attenuation and surrounded by the attenuation peaks.

5G MMWave communication system; total gaseous atmospheric attenuation is calculated by having equiprobability summing. The total MMWave atmospheric losses for 5G MMWave is calculated by having sum of equation (2) and (4).

$$
A_{\text {gas }}=A_{\text {water }}+A_{\text {oxygen }}
$$

As per equations (2) and (4) summation to the equation (5) the total atmospheric attenuation due to the MMWave water vapor and oxygen has been plotted with mmWave frequencies in the Fig. (3). In this Fig. (3) logarithmic scale has been used with the MMWave frequency range $10 \mathrm{GHz}$ to $1000 \mathrm{GHz}$. This plot provides all peaks in a single graph to decide transmission window easily.

It is easy to design and select MMWave frequency for Delhi based 5G MMWave communication system. The minimal attenuation area in Fig. (3) shows the space for transmission with minimal attenuation due to atmosphere. Table (1) depicts the attenuation due to water vapor, oxygen and total gaseous attenuation for the mmWave frequencies as per table (1):

Table 1. Atmospheric Gaseous Attenuation Values for 5G mmWave Wireless Communication System

\begin{tabular}{|c|c|c|c|}
\hline Attenuation dB & $28 \mathrm{GHz}$ & $37 \mathrm{GHz}$ & $39 \mathrm{GHz}$ \\
\hline water & 0.63 & 0.646 & 0.53 \\
\hline Oxygen & 0.016 & 0.646 & 0.019 \\
\hline Gaseous / Total & 0.646 & 0.646 & 0.549 \\
\hline
\end{tabular}

\section{Atmospheric Rain \& Fog Attenuation}

Atmospheric attenuation has one of the major factor due to rain and rain drops play major role for atmospheric attenuation. Rain drops acts as a poor dielectric and absorbs the MMWave power and dissipates the MMWave signal power by scattering or heat loss. Here we are using modified ITU-R rain attenuation model to calculate MMWave rain attenuation majorly based on rain rate. Atmospheric specific attenuation for 5G MMWave communication link is given equation (6) in $\mathrm{db} / \mathrm{km}$ [19-23]:

$$
\Gamma_{R}=k R^{a}
$$

In equation (6) $\mathrm{R}$ is the rain rate in $\mathrm{mm} / \mathrm{hr}$ and $\mathrm{k}$ and alpha are frequency and polarization dependent coefficients which are detailed in the prior literature. Total rain attenuation rain A exceeded for $0.01 \%$ of an year in $\$ \mathrm{~dB} \$$ is shown by equation (7)

$$
A_{\text {rain }}=\gamma_{R} L_{s} r_{0.01}
$$

Fig. (4), (5), (6) and (7) show the summary of the rain attenuation graphs for the coefficients k, alpha, specific attenuation and total rain attenuation graph of Delhi atmospheric rain attenuation. 5G MMWave communication system; attenuation due to rain for the frequencies $28 \mathrm{GHz}, 37 \mathrm{GHz}$ and $39 \mathrm{GHz}$ are marked the figures. It is clear from the plot that rain attenuation keeps increasing with frequency. 
MMWave 5G MMWave communication system also suffers with the fog attenuation. in the presence of fog goes through significant amount of attenuation. Atmospheric fog attenuation has dependent parameters like amount of water, temperature, fog particle average size, water distribution and etc. Attenuation due to fog is given by equation (8)

$$
\begin{aligned}
& A_{f o g}=L_{s} \gamma_{c} \\
& A_{f o g}=L_{s} K_{l} M
\end{aligned}
$$

In Delhi; MMWave 5G MMWave communication system will suffer fog attenuation in the month of December and January. Fig. (8) plots the attenuation due to fog for different temperatures. It is clear that the fog attenuation is significant value for the frequencies $28 \mathrm{GHz}, 37 \mathrm{GHz}$ and $39 \mathrm{GHz}$. Plot also shows fog attenuation also depends on the varying temperatures.

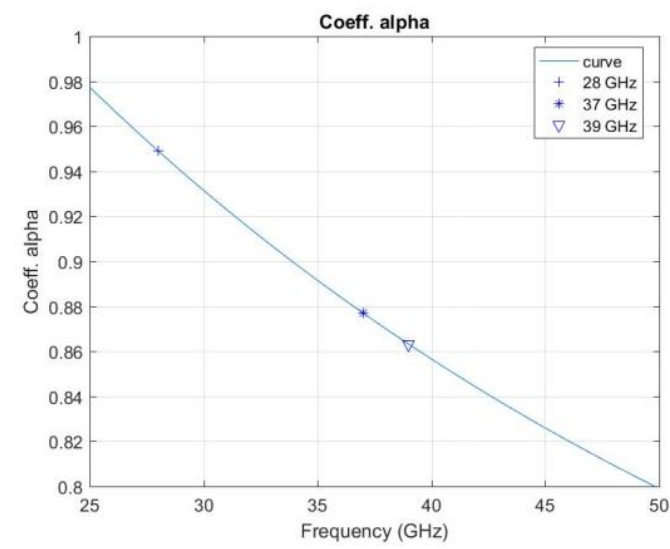

Fig.4. Atmospheric Rain Attenuation Coefficient Alpha for 5G mmWave Wireless Communication System

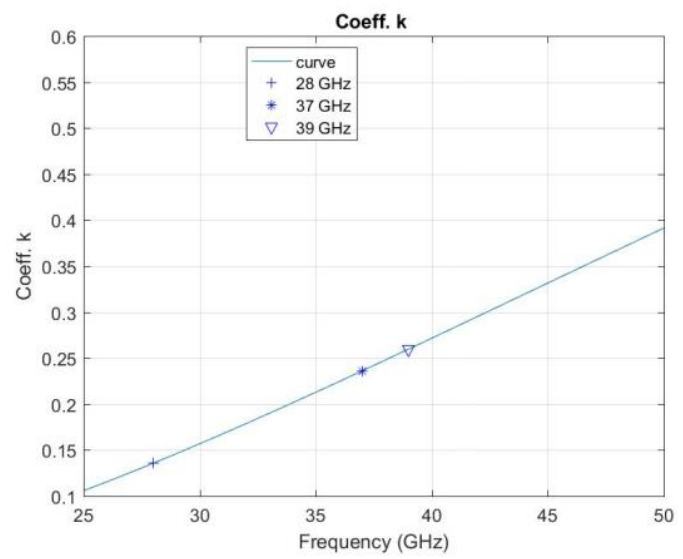

Fig.5. Atmospheric Rain Attenuation Coefficient k for 5G mmWave Wireless Communication System 


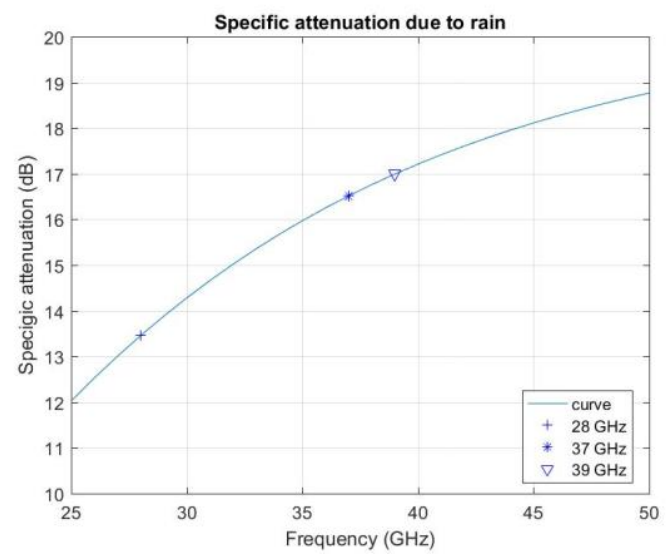

Fig.6. Atmospheric Rain Attenuation Specific for 5G mmWave Wireless Communication System

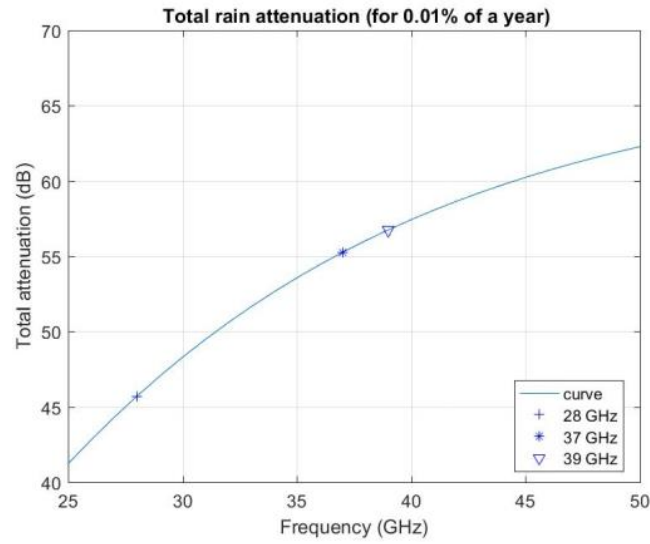

Fig.7. Atmospheric Rain Attenuation for 5G mmWave Wireless Communication System

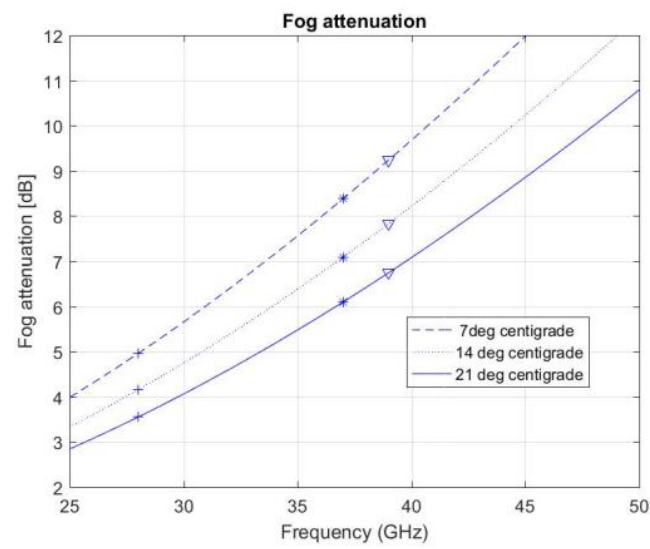

Fig.8. Atmospheric Fog Attenuation for 5G mmWave Wireless Communication System 


\section{Software Defined Channel Capacity}

Software Defined MMWave facilitates transmission power control in a real manner. 5G millimeter wave communication system transmitter and receivers can be controlled by artificial intelligent (AI) software defined (SD) mmwave communication system. AI based software defined MMWave facilitates methods to control the transceiver as per atmospheric attenuation [19-23].

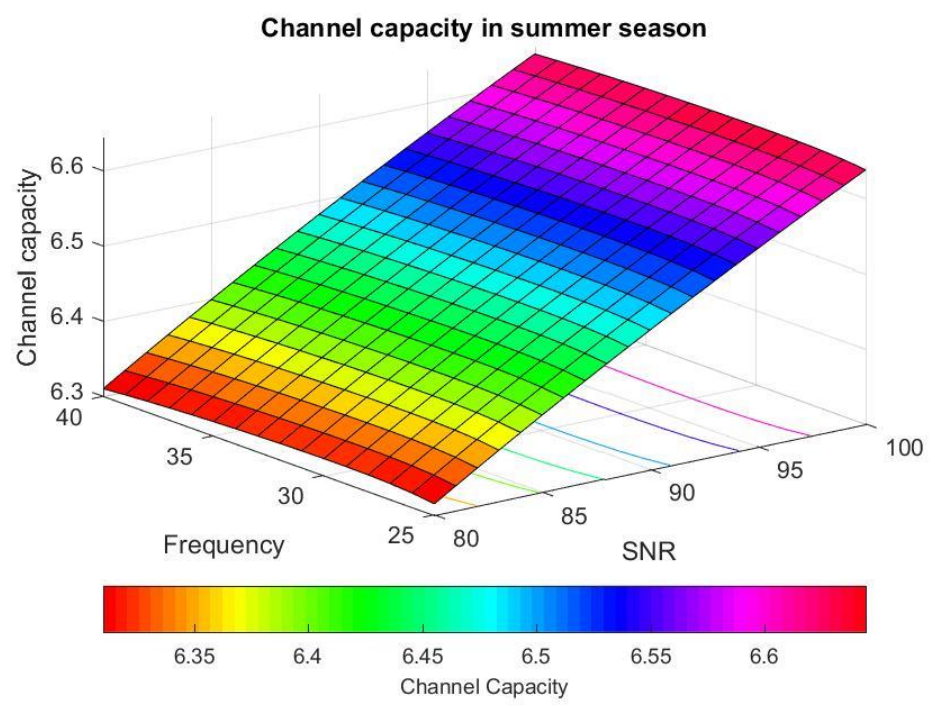

Fig.9. Channel Capacity in Summer Season for 5G mmWave Wireless Communication System

In this paper intelligent adaptive transmitter based on trend of the atmospheric conditions tunes to machine learning (ML) based derivation of channel capacity. The ML based transmitter is a supervised ML device and it has provision of self teaching learning machine based on data. Here intelligent an adaptive transmitter which is having machine learning (ML) based on previous trends of the attenuations decides the optimal channel capacity. Its intelligence is based on the various type of attenuations as per various conditions as described. The decision-directed adaptive receiver has the same structure as the gremlin receiver, but uses its own decisions . The adaptive ML based transmitter is useful to intelligently provide the required channel capacity. It intelligently regulates the demand and need of channel capacity in 5G millimeter wave communication system in Delhi. Shannon channel capacity in the presence of continuous additive white Gaussian noise channel is given by equation (9) [19-26]. 


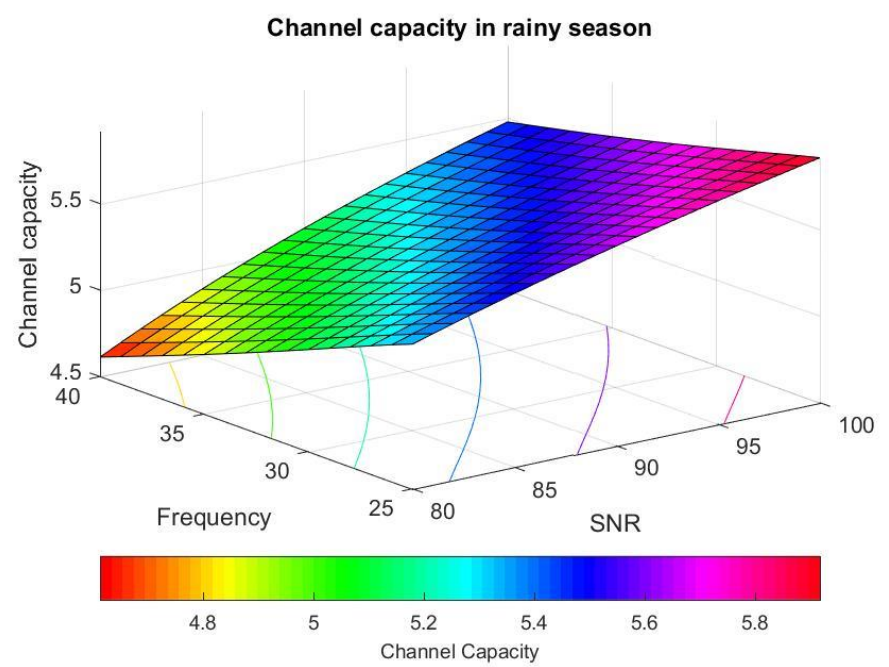

Fig.10. Channel Capacity in Rainy Season for 5G mmWave Wireless Communication System

$$
\frac{C}{B}=\log 2\left(1+\frac{S}{N}\right)
$$

The received signal-to-noise power ratio $(\mathrm{S} / \mathrm{N})$ in $\mathrm{dB}$ due to water vapor, oxygen, rain and fog are classified using machine learning (ML) for various seasons. Delhi summer season includes atmospheric attenuation due to atmospheric water vapor and oxygen. For this city rainy season includes atmospheric attenuation due to atmospheric water vapor, rain and oxygen. Further, in winter season fog play one of the role for attenuation and winter season includes atmospheric attenuation due to atmospheric water vapor, fog and oxygen.

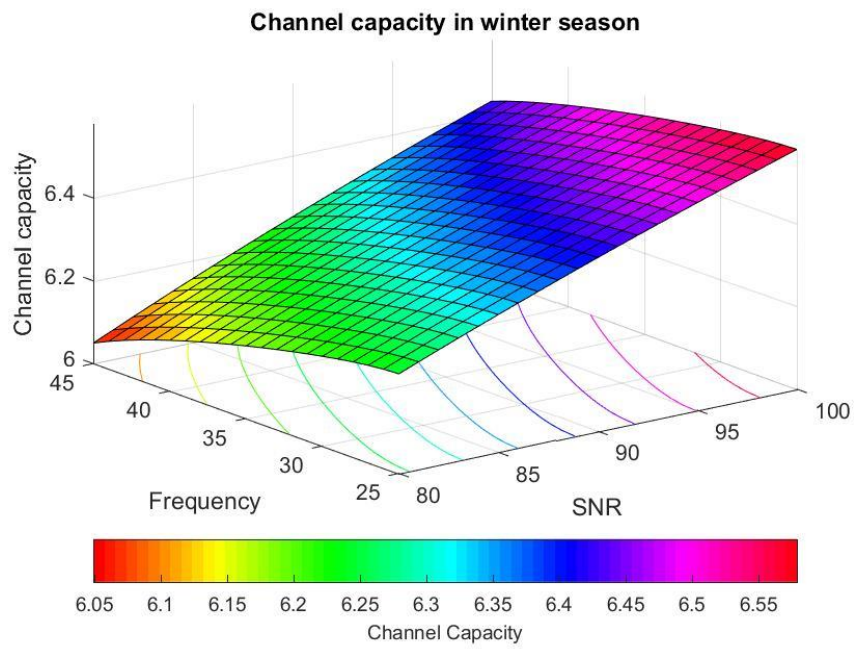

Fig.11. Channel Capacity in Winter Season for 5G mmWave Wireless Communication System 
In Delhi; MMWave 5G MMWave communication system software defined channel capacity are shown for summer, rain and winter in Fig. (9), (10) and (11). These plots are shown with channel capacity as per atmosphere and shown for the frequencies $28 \mathrm{GHz}, 37 \mathrm{GHz}$ and $39 \mathrm{GHz}$. By using AI based software controlled transmitter and or receivers the channel capacity can be controlled intelligently.

\section{Conclusions}

This paper presented atmospheric attenuation calculation for various atmosphere attenuation causes for $5 \mathrm{G}$ millimeter wave propagation attenuation at the frequencies $28 \mathrm{GHz}, 37 \mathrm{GHz}$ and $39 \mathrm{GHz}$ for Delhi (India). It is shown in the paper that the atmospheric attenuation due to water vapor, oxygen, rain and fog varies and depends on the millimeter wave frequencies. This research also signifies the reason of the selection of the frequencies $28 \mathrm{GHz}, 37 \mathrm{GHz}$ and $39 \mathrm{GHz}$ for 5G Millimeter Wave (mmWave) Communication System. These frequencies $28 \mathrm{GHz}, 37 \mathrm{GHz}$ and $39 \mathrm{GHz}$ for 5G Millimeter Wave (mmWave) Communication System shows minimal attenuation.

Software Defined MMWave facilitates transmission power control in a real manner. 5G millimeter wave communication system transmitter and receivers can be controlled by the software defined mmwave communication system. Software Defined MMWave facilitates methods to control the transceiver as per atmospheric attenuation and demand and need of channel capacity in 5G millimeter wave communication system. Here intelligent an adaptive transmitter which is having machine learning (ML) based on previous trends of the attenuations decides the optimal channel capacity. Its intelligence is based on the various type of attenuations as per various conditions as described. The decision-directed adaptive receiver has the same structure as the gremlin receiver, but uses its own decisions. The adaptive ML based transmitter is useful to intelligently provide the required channel capacity.

This research paper is useful for transmission link budget for 5G Millimeter Wave (mmWave) Communication system for instant installation. Future work involves the identifying the various other dynamic atmospheric attenuation causes for the atmospheric attenuation for 5G Millimeter Wave (mmWave) Communication.

\section{Acknowledgements}

This paper is specifically meant for educational purpose for completing Ph.D. degree with Delhi Technological University (DTU), Delhi India. This paper is prepared for submission to under Ph.D. progress. The authors are very much thankful to Samsung Research Institute Noida, India and Delhi Technological University (DTU) earlier recognized as Delhi-College of Engineering (DCE), New Delhi, India.

\section{References}

[1] Ghosh, Amitabha. "Can mmwave wireless technology meet the future capacity crunch?." Presentation at IEEE ICC (2013).

[2] Bergren, Steven. "Design Considerations for a 5G Network Architecture." arXiv preprint arXiv:1705.02902 (2017).

[3] Agrawal, S. K., and Kapil Sharma. "5G millimeter wave (mmWave) communications." Computing for Sustainable Global Development (INDIACom), 2016 3rd International Conference on. IEEE, 2016.

[4] Sun, Shu, et al. "Investigation of prediction accuracy, sensitivity, and parameter stability of large-scale propagation path loss models for 5G wireless communications." IEEE Transactions on Vehicular Technology 65.5 (2016): 2843-2860. 
[5] Agrawal, S. K., and Kapil Sharma. "5G Millimeter Wave (mmWave) Communication System with Software Defined Radio (SDR)." International Conference on Recent Trends in Engineering \& Science (ICRTES-16), 29th-30th September. 2016.

[6] Zhouye Pi et al., "An Introduction to Millimeter- Wave Mobile Broadband Systems, " IEEE Communications Magazine, vol.49, no.6, pp.101- 107, Jun. 2011.

[7] Andrews J.G. et al., "What Will 5G Be?, " IEEE JSAC Special Issue On 5G Wireless Communication Systems, May 2014.

[8] Yang L-L, Hanzo L. Software-defined-radio-assisted adaptive broadband frequency hopping multicarrier DSCDMA. IEEE Commun Mag 2002;40(3):174-83.

[9] Scudder, II. J., Adaptive communication receivers. IEEE Trans. Inform. Theory, 1965, 2, 167-174

[10] X. Wang, X. Li, and V. C. M. Leung, "Artificial Intelligence-Based Techniques for Emerging Heterogeneous Network: State of the Arts, Opportunities, and Challenges," IEEE Access, vol. 3, 2015, pp. 1379-91.

[11] G. Huang, G.-B. Huang, S. Song, and K. You, "Trends in extreme learning machines: A review," Neural Netw., vol. 61, pp. 32_48, Jan. 2015.

[12] Theodore S. Rappaport et al., "Millimeter Wave Mobile Communications for 5G Cellular: It Will Work!," IEEE Access, vol.1, pp.335-349, May 2013.

[13] FCC, "Millimeter wave Propagation: Spectrum management Implications," office of Eng. and Tech., Bulletin no. 70, July, 1997.

[14] FCC "Report And Order And Further Notice Of Proposed Rulemaking”, FCC 16-89, July, 2016

[15] Evaluating Compliance with FCC Guidelines for Human Exposure to Radiofrequency Electromagnetic Fields, Federal Communication Commission, OET Bulletin 65, Edition 97-01.

[16] ITU Recommendation ITU-R RPN.618-4, 1996

[17] International Telecommunication Union, "Water Vapour: Surface Density and Total Columnar Content," Recommendation ITU-R P.836-3, Electronic Publication, Geneva, Switzerland, November 2001.

[18] Recommendation, “Attenuation by atmospheric gases," in ITU-R P.676-8, 102009.

[19] S. K. Agrawal and P. Garg, "Calculation of Channel Capacity Considering the Effect of Different Seasons for Higher Altitude Platform System", Wireless Personal Communications, Springer, Wireless Personal Communications: vol. pp-719-729, Issue 42010.

[20] S. K. Agrawal and P. Garg, "Calculation of the Channel Capacity in the Presence of Vegetation and Urban-site Environment for Higher Altitude Platform Communication System", to Wireless Personal Communications", IET Microwaves, Antenna \& Propagation, IET Microw. Antennas Propag., Vol. 3, Iss. 4, pp. 703-713, 2009.

[21] Agrawal, S. K., and Kapil Sharma. " Software Defined Radio Based Channel Capacity In 5G Millimeter Wave Communication System" International Virtual Conference on Computer Science, Engineering and Technology (25th - 28th December, 2016), Indore - India.

[22] S. K. Agrawal and K. Sharma, "5G Millimeter Wave (mmWave) Communication System with Software Defined Radio (SDR)", International Journal for Innovative Research in Science \& Technology, Volume 2, Issue 9, September 2016

[23] Dissanayake, A., Allnutt, J., \& Haidara, F. (1997). A prediction model that combines rain attenuation and other propagation impairments along earth-satellite paths. In Proceedings of IEEE Transactions on Antenna and Propagation (Vol. 45, pp. 1546-1558), Oct. 1997.

[24] Blum, Avrim L., and Pat Langley. "Selection of relevant features and examples in machine learning." Artificial intelligence 97.1-2 (1997): 245-271.

[25] Kotsiantis, Sotiris B., I. Zaharakis, and P. Pintelas. "Supervised machine learning: A review of classification techniques." Emerging artificial intelligence applications in computer engineering 160 (2007): 3-24. 
[26] J. Mitola. III, “Technical Challenges in the Globalization of Software Radio," IEEE Commun. Mag., Feb. 1999, pp. 84-89.

\section{Authors' Profiles}

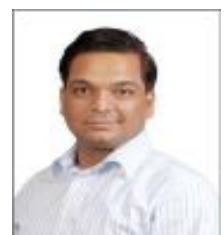

Mr. S. K. Agrawal's educational and professional interest include 5G, Antenna, Beam, RF, Wireless communication, Networking, Channel Capacity, MIMO, Physical Layer, Machine Learning (ML), Artificial Intelligence (AI) and related technologies. He has published various research papers in international conferences and journals in the domain of wireless communications and intelligent solutions along with best paper award. He has invented several novel features which are protected as several national and international patents. He is member of TPCs ( international conferences/ journal), Member of Indian Science Congress Association (ISCA), Member of International Computer Science and Engineering Society (ICSES), International Association of Engineers (IAENG), IAENG Society of Artificial Intelligence (AI) and etc.

S. K. Agrawal has reviewed various international research papers for conferences and journals in the domain of mobile communications. He is currently working for Research / Inventions / Patents in Samsung R\&D, Noida India. He has extensive experience in research and intellectual property (IP) domain. Agrawal is currently pursuing Ph.D. in Artificial Intelligent (AI) wireless solutions from Delhi Technological University (DTU), formerly known as Delhi College of Engineering (DCE) Delhi. He is having Masters degree in Signal processing (SP) from Netaji Subhas Institute of Technology (Formerly: Delhi Institute of Technology), University of Delhi, India. He has worked as a researcher at Telecommunications Research Centre (TRC), University of Limerick (UL) Ireland and Indian Institute of Technology (IIT) Delhi. He has also worked with Computer Patent Annuities (CPA) Global, National Atmospheric Research Laboratory (NARL), Department of Space, (Govt. of India) India and ASC Zee Networks, DTH R\&D India.

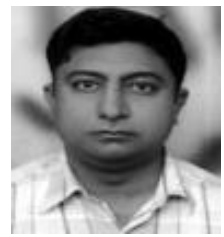

Dr. Kapil Sharma was born in Haryana, India. In 2011, he has completed Doctors Degree in Computer Science and Engineering under the Faculty of Engineering and Technology at the M. D. University, Rohtak (Haryana), India. He has obtained his Bachelor of Engineering and Master of Technology Degrees in Computer Science \& Engineering and Information Technology from M. D. University, Rohtak and IASE, Rajasthan, India in 2000 and 2005 respectively. He has published various research papers in international conferences and journals in the domain of mobile communications along with several national and international patents.

$\mathrm{He}$ is head of department of IT department and also Associate Professor in Department of Computer Engineering; Delhi Technological University. He previously worked as Assistant Professor with School of Information Communication \& Technology; Department of Computer Science \& Engineering; Greater Noida, Gautam Budh Nagar and as Reader and Head with Department of Information Technology; Guru Premsukh Memorial College of Engineering 245, Budhpur Villege, G.T Karnal Road; Delhi.

How to cite this paper: S. K. Agrawal, Kapil Sharma," Intelligent Software Defined Atmospheric Effect Processing for 5th Generation (5G) Millimeter Wave (MMWave) Communication System", International Journal of Wireless and Microwave Technologies(IJWMT), Vol.8, No.2, pp. 15-26, 2018.DOI: 10.5815/ijwmt.2018.02.02 